09

\title{
Люминесцентные свойства в видимом и коротковолновом ИК-спектральных диапазонах апатитов $\mathrm{Sr}_{2} \mathrm{La}_{8-x} \mathrm{Tm}_{x}\left(\mathrm{GeO}_{4}\right)_{6} \mathrm{O}_{2}$ $(x=0.1-1.0)$
}

\author{
(С) Я.В. Бакланова, О.А. Липина, Л.Л. Сурат, А.Ю. Чуфаров, А.П. Тютюнник, В.Г. Зубков \\ Институт химии твердого тела УрО РАН, \\ Екатеринбург, Россия \\ E-mail: baklanovay@ihim.uran.ru
}

Поступила в Редакцию 2 марта 2020 г.

В окончательной редакции 2 марта 2020 г.

Принята к публикации 24 марта 2020 г.

\begin{abstract}
Впервые твердофазным методом синтезированы твердые растворы $\mathrm{Sr}_{2} \mathrm{La}_{8-x} \operatorname{Tm}_{x}\left(\mathrm{GeO}_{4}\right)_{6} \mathrm{O}_{2}(x=0.1-1.0)$ со структурой апатита, исследованы спектрально- люминесцентные свойства и продемонстрирована перспективность использования данных соединений в качестве люминофоров видимого и коротковолнового инфракрасного диапазонов. Люминесценция германатов $\mathrm{Sr}_{2} \mathrm{La}_{8-x} \mathrm{Tm}_{x}\left(\mathrm{GeO}_{4}\right)_{6} \mathrm{O}_{2}$, возникающая под воздействием ультрафиолетового излучения, характеризуется высокой чистотой голубого цвета и близкими к коммерчески доступным люминофорам значениями координат цветности. Помимо этого, показано, что соединения $\mathrm{Sr}_{2} \mathrm{La}_{8-x} \mathrm{Tm}_{x}\left(\mathrm{GeO}_{4}\right)_{6} \mathrm{O}_{2}$ эффективно преобразуют лазерное излучение с длиной волны $808 \mathrm{~nm}$ в серию эмиссионных линий в спектральном диапазоне $1.3-2.2 \mu \mathrm{m}$, обусловленных последовательными переходами ${ }^{3} \mathrm{H}_{4} \rightarrow{ }^{3} \mathrm{~F} 4$ и ${ }^{3} \mathrm{~F}_{4} \rightarrow{ }^{3} \mathrm{H}_{6}$ в ионах $\mathrm{Tm}^{3+}$. Германат $\mathrm{Sr}_{2} \mathrm{La}_{7.6} \mathrm{Tm}_{0.4}\left(\mathrm{GeO}_{4}\right)_{6} \mathrm{O}_{2}$ с максимальной интенсивностью эмиссии в коротковолновой инфракрасной области демонстрирует высокую термическую стабильность люминесценции в интервале $30-220^{\circ} \mathrm{C}$.
\end{abstract}

Ключевые слова: германат, апатит, люминесценция, тулий, коротковолновый ИК-диапазон.

DOI: $10.21883 /$ FTT.2020.08.49610.045

\section{1. Введение}

В последние несколько десятилетий большое внимание уделяется поиску материалов с интенсивной эмиссией в коротковолновом и среднем инфракрасном (ИК) диапазоне для различных биомедицинских и инфокоммуникационных приложений [1-3]. К настоящему времени наиболее исследованными с этой точки зрения классами неорганических соединений являются теллуриты, фосфаты, алюминаты и значительно в меньшей степени силикаты и германаты, допированные различными лантаноидами [4-8]. Оптические среды, рассматриваемые для применений в ИК-диапазоне, должны обладать рядом обязательных характеристик, такими, например, как высокая химическая стабильность, прозрачность в широком диапазоне длин волн и относительно низкие значения фононной энергии. Вышеперечисленное уменьшает вероятность протекания безызлучательных процессов и, соответственно, способствует увеличению эффективности люминесценции [8]. К числу таких сред могут быть отнесены соединения со структурой апатита (пространственная группа $P 6_{3} / m, Z=1$ ) и обобщенной формулой $M_{10}\left(X_{4}\right)_{6} Z_{2}$, где $M-\mathrm{Li}^{+}-\mathrm{Cs}^{+}, \mathrm{Mg}^{2+}-\mathrm{Sr}^{2+}$ и лантаноиды, $X-\mathrm{Si}^{4+}, \mathrm{Ge}^{4+}, \mathrm{P}^{5+}, \mathrm{V}^{5+} ; Z-\mathrm{O}^{2-}, \mathrm{Cl}^{-}$, $\mathrm{F}^{-}, \mathrm{OH}-$ и др. $[9,10]$. Кристаллическая решетка апатитов построена на основе комбинации катионов, расположенных внутри трехшапочных тригональных призм $\left(M \mathrm{O}_{9}\right)$, тетраэдров $\left(\mathrm{XO}_{4}\right)$ и пятиугольных пирамид $\left(M 2 \mathrm{O}_{7}\right)$. Вдоль оси с расположены гексагональные туннели, об- разованные атомами $\left(M_{2}\right)_{6} Z_{2}$. Комбинация различных катионов и анионов позволяет создавать множество композиций со своими уникальными свойствами, нашедшими применения не только в качестве люминофоров с эмиссией в видимом спектральном диапазоне, но и в качестве биологически совместимых препаратов, твердых электролитов, катализаторов и т.д. [11-14]. В последнее время существенное внимание научного сообщества уделено исследованиям фотолюминесцентных характеристик силикатов и германатов со структурой апатита при down-конверсии возбуждающего излучения [15-20]. Работ, затрагивающих перспективы применения данных соединений для ИК-приложений, значительно меньше. Тем не менее ранее была показана возможность использования составов на основе кремниевых и германиевых апатитов, активированных $\mathrm{Nd}^{3+}, \mathrm{Yb}^{3+}, \mathrm{Nd}^{3+} / \mathrm{Ho}^{3+}$, $\mathrm{Ho}^{3+} / \mathrm{Er}^{3+} / \mathrm{Tm}^{3+}$ в качестве активных сред для твердотельных лазеров и люминофоров ближнего и коротковолнового ИК-диапазонов [21-24]. Наиболее эффективными активаторами для получения интенсивной эмиссии в интервале $1-3 \mu \mathrm{m}$ являются ионы с развитой системой мультиплетов, такие как $\mathrm{Dy}^{3+}, \mathrm{Ho}^{3+}, \mathrm{Er}^{3+}$ и $\mathrm{Tm}^{3+}$. Использование иона тулия в качестве активатора имеет ряд преимуществ по сравнению с вышеперечисленными лантаноидами: возможность применения в качестве источника оптической накачки коммерческих лазерных диодов с длиной волны излучения $808 \mathrm{~nm}$ без введения в оптическую матрицу сенсибилизатора (иона $\mathrm{Nd}^{3+}$ ) и получение интенсивной эмиссии, охватывающей 
широкий ИК-диапазон от $1.3 \mu \mathrm{m}$ до $2.7 \mu \mathrm{m}\left({ }^{3} F_{4} \rightarrow{ }^{3} H_{4}\right.$, ${ }^{3} \mathrm{H}_{4} \rightarrow{ }^{3} \mathrm{H}_{6}$ и ${ }^{3} \mathrm{~F}_{4} \rightarrow{ }^{3} \mathrm{H}_{5}$ переходы иона $\left.\mathrm{Tm}^{3+}\right)$ [3,8]. В данной работе приведены исследования эмиссионных свойств люминофоров коротковолнового ИК-диапазона на основе $\mathrm{Sr}_{2} \mathrm{La}_{8}\left(\mathrm{GeO}_{4}\right)_{6} \mathrm{O}_{2}: \mathrm{Tm}^{3+}$ со структурой апатита. Отдельное внимание уделено изучению концентрационных зависимостей интенсивности ИК-люминесценции, ее стабильности при повышенных температурах и определению механизма термического тушения. Более того, поскольку ион тулия традиционно используется для генерации свечения в „синей“ области спектра с целью получения люминофоров белого цвета $[15,16,25,26]$, в том числе и в соединениях со структурой апатита [19], нами были дополнительно обсуждены люминесцентный свойства германатов $\mathrm{Sr}_{2} \mathrm{La}_{8}\left(\mathrm{GeO}_{4}\right)_{6} \mathrm{O}_{2}: \mathrm{Tm}^{3+}$ в видимом спектрально диапазоне при возбуждении ультрафиолетовым излучением.

\section{2. Методика эксперимента}

Германаты $\mathrm{Sr}_{2} \mathrm{La}_{8-x} \mathrm{Tm}_{x}\left(\mathrm{GeO}_{4}\right)_{6} \mathrm{O}_{2}(x=0.1-1.0)$ были получены твердофазным методом. В качестве исходных реагентов использовали $\mathrm{La}_{2} \mathrm{O}_{3}(99.99 \%)$ и $\mathrm{Tm}_{2} \mathrm{O}_{3}$ (99.99\%), предварительно отожженные при $900^{\circ} \mathrm{C}$ в течение $5 \mathrm{~h}, \mathrm{SrCO}_{3}(99.9 \%)$ с $5 \%$ избытком и $\mathrm{GeO}_{2}$ (99.95\%), предварительно отожженные при $650^{\circ} \mathrm{C}$ в течение $5 \mathrm{~h}$. Смесь исходных компонентов, тщательно перетирали и отжигали при температуре $700-750^{\circ} \mathrm{C}$ в течение $15 \mathrm{~h}$. Далее образцы последовательно отжигали при температурах $800-1200^{\circ} \mathrm{C}$ с шагом 100 градусов и выдержкой в течение $20 \mathrm{~h}$, охлаждением и перетиранием на воздухе.

Рентгенофазовый анализ синтезированных соединений осуществляли с помощью дифрактометра STADI-P (STOE), оснащенного линейным позиционно-чувствительным детектором. Съемка проводилась в $\mathrm{Cu} K_{\alpha 1}$ излучении в интервале углов $2 \theta 5-120^{\circ}$ с шагом $0.02^{\circ}$. В качестве внешнего стандарта использовали поликристаллический кремний с параметром элементарной ячейки $a=5.43075(5) \AA ̊$. Идентификация фаз проведена с использованием картотеки PDF2 (ICDD, 2016). Уточнение кристаллических структур соединений проведено методом полнопрофильного анализа Ритвельда с использованием программного пакета GSAS [27,28]. Профили рефлексов описывались с использованием функции псевдо-Войта с учетом угловой зависимости ширины пика. При описании фона применяли полином Чебышева. В качестве исходной модели для уточнения профиля рентгенограмм синтезированных составов использовались данные кристаллической структуры $\mathrm{Sr}_{2} \mathrm{La}_{8}\left(\mathrm{GeO}_{4}\right)_{6} \mathrm{O}_{2}$ (FIZ ICSD card № 418709).

Спектры фотолюминесценции были получены с использованием флуоресцентного спектрофотометра Cary Eclipse (Varian), оборудованного импульсной ксеноновой лампой мощностью $75 \mathrm{~kW}$ (длина импульса $2 \mu \mathrm{s}$; частота импульсов $80 \mathrm{~Hz}$; разрешение по длинам волн $0.5 \mathrm{~nm}$;
ФЭУ Hamamatsu R928). Спектры люминесценции в диапазоне длин волн от $1.3 \mu \mathrm{m}$ до $2.2 \mu \mathrm{m}$ получены методом синхронной съемки с использованием монохроматора МДР-204 (90 геометрия; дифракционная решетка 600 lines/mm; оптический затвор, осуществляющий модуляцию с частотой $200 \mathrm{~Hz}$ ) и $\mathrm{PbS}$ фотосопротивления (ЛОМО-Фотоника). В качестве внешнего источника возбуждения использовался диодный лазер мощностью 140 $\mathrm{mW}$, длина волны излучения $808 \mathrm{~nm}$ (KLM-H808-120-5, ФТИ-Оптроник). Мощность лазерного излучения контролировали детектором 11XLP12-3S-H2 (Standa). Кopректировку спектров на спектральную чувствительность детектора не проводили. Кинетические кривые затухания люминесценции, измеренные для возбужденных состояний тулия ${ }^{3} H_{4}$ и ${ }^{3} F_{4}$ регистрировали с использованием охлаждаемого фотоумножителя Н10330С-75 (Hamamatsu) с фотокатодом из InP/InGaAs и осциллографа TDS-3052 (Tektronix). В качестве внешнего источника излучения использовался импульсный лазер с длиной волны возбуждения $263 \mathrm{~nm}$ (длительность импульсов $5 \mathrm{~ns}$ при частоте повторения $200 \mathrm{~Hz}, 4-я$ гармоника DPSS Nd лазера Tech 263, Laser-export). Вышеупомянутые измерения были выполнены при комнатной температуре. Спектры люминесценции в диапазоне длин волн от $1.3 \mu \mathrm{m}$ до $2.2 \mu \mathrm{m}$ и кривые затухания люминесценции, измеренные для возбужденных состояний тулия, также регистрировали в интервале температур от $30^{\circ} \mathrm{C}$ до $220^{\circ} \mathrm{C}$ при использовании температурно-контролируемой ячейки GS-21525 (Specac Ltd).

\section{3. Экспериментальные результаты и их обсуждение}

Анализ данных порошковой рентгеновской дифракции составов $\mathrm{Sr}_{2} \mathrm{La}_{8-x} \mathrm{Tm}_{x}\left(\mathrm{GeO}_{4}\right)_{6} \mathrm{O}_{2}(x=0.1-1.0)$ показал, что все рефлексы на дифрактограммах твердых растворов соответствуют апатиту $\mathrm{Sr}_{2} \mathrm{La}_{8}\left(\mathrm{GeO}_{4}\right)_{6} \mathrm{O}_{2}$ (FIZ ICSD card № 418709), дополнительных рефлексов от промежуточных фаз или примесей, типичных для твердофазного синтеза, таких как германат лантана $\mathrm{La}_{4} \mathrm{Ge}_{4} \mathrm{O}_{14}$, оксиды лантана $\mathrm{La}_{2} \mathrm{O}_{3}$ и тулия $\mathrm{Tm}_{2} \mathrm{O}_{3}$ не обнаружено. Рентгенограммы $\mathrm{Sr}_{2} \mathrm{La}_{8-x} \mathrm{Tm}_{x}\left(\mathrm{GeO}_{4}\right)_{6} \mathrm{O}_{2} \quad(x=0.1-1.0)$ проиндицированы в гексагональной сингонии (пространственная группа $\left.P 6_{3} / m, Z=1\right)$. Структура гексагонального апатита $\mathrm{Sr}_{2} \mathrm{La}_{8}\left(\mathrm{GeO}_{4}\right)_{6} \mathrm{O}_{2}$ состоит из трехшапочных тригональных призм ( $\mathrm{Sr} / \mathrm{La} 1) \mathrm{O}_{9}$, пятиугольных пирамид $\mathrm{La}_{2} \mathrm{O}_{7}$ и тетраэдров $\mathrm{GeO}_{4}$. Сочлененные по ребрам пирамиды $\mathrm{La}_{2} \mathrm{O}_{7}$ образуют каналы вдоль оси с, которые между собой соединены тетраэдрами $\mathrm{GeO}_{4}$. Большие гексагональные пустоты между каналами $\mathrm{La}_{2} \mathrm{O}_{7}$ заняты полиэдрами $(\mathrm{Sr} / \mathrm{La} 1) \mathrm{O}_{9}$, соединенными гранями, причем позиции $\mathrm{Sr} / \mathrm{La} 1$ заселена в равных долях атомами стронция и лантана. Кристаллические параметры твердых растворов приведены в таблице. При замещении ионов $\mathrm{La}^{3+}\left(C R_{\mathrm{IX}}\left(\mathrm{La}^{3+}\right)=1.356 \AA\right)$ ионами $\mathrm{Tm}^{3+}$ 
Параметры кристаллических решеток твердых растворов $\operatorname{Sr}_{2} \mathrm{La}_{8-x} \operatorname{Tm}_{x}\left(\mathrm{GeO}_{4}\right)_{6} \mathrm{O}_{2}(x=0.1-1.0)$ (пространственная группа $P 6_{3} / m$, $Z=1)$

\begin{tabular}{c|l|l|l|l|l|l|l|l}
\hline$X$ & $a, \AA$ & $c, \AA$ & $V, \AA^{3}$ & $D_{x}, \mathrm{~g} / \mathrm{sm}^{3}$ & $R_{p}[\mathrm{C}, \%$ & $\chi^{2}$ & $R\left(F^{2}\right), \%$ \\
\hline 0.100 & $9.90494(12)$ & $7.31619(11)$ & $621.61(1)$ & 5.711 & 4.5 & 1.03 & 3.87 \\
0.125 & $9.90270(8)$ & $7.31487(7)$ & $621.22(1)$ & 5.720 & 3.2 & 1.218 & 2.95 \\
0.150 & $9.90064(8)$ & $7.31146(7)$ & $620.67(1)$ & 5.727 & 3.56 & 1.009 & 3.11 \\
0.200 & $9.89656(9)$ & $7.30280(8)$ & $619.42(1)$ & 5.744 & 3.97 & 0.9899 & 3.18 \\
0.400 & $9.89482(12)$ & $7.29334(11)$ & $618.40(1)$ & 5.768 & 4.37 & 1.02 & 3.91 \\
0.600 & $9.88698(12)$ & $7.27635(10)$ & $615.99(1)$ & 5.806 & 4.49 & 1.056 & 3.74 \\
0.800 & $9.88686(9)$ & $7.27362(8)$ & $615.74(1)$ & 5.827 & 3.79 & 1.079 & 3.04 \\
1.000 & $9.87831(9)$ & $7.25658(8)$ & $613.24(1)$ & 5.864 & 3.89 & 1.074 & 3.47
\end{tabular}

$\left(C R_{\mathrm{IX}}\left(\mathrm{Tm}^{3+}\right)=1.192 \AA\right)[29,30]$ наблюдается уменьшение параметров и объема элементарной ячейки. Немонотонность изменения параметров может быть объяснена происходящим при изменении концентрации перераспределением тулия между двумя кристаллографическими позициями - центров тригональных призм $(4 f)$ и пятиугольных пирамид $(6 h)$ с симметрией $C_{3}$ и $C_{s}$, соответственно.

Спектры люминесценции $\mathrm{Sr}_{2} \mathrm{La}_{8-x} \mathrm{Tm}_{x}\left(\mathrm{GeO}_{4}\right)_{6} \mathrm{O}_{2}$ $(x=0.1-1.0)$ в диапазоне $430-830 \mathrm{~nm}$ имеют типичный профиль с четырьмя хорошо узнаваемыми линиями, обусловленными $4 f-4 f$ переходами в ионах $\mathrm{Tm}^{3+}[16,19,31]$ (рис. 1). Выбор схемы накачки при $\lambda_{\mathrm{ex}}=356 \mathrm{~nm}$ (переход ${ }^{3} H_{6} \rightarrow{ }^{31} D_{2}$ в ионах $1 \mathrm{gTm}^{3+}$ ) (вставка к рис. 1) основан на существовании многочисленных коммерческих источников, доступных на данной длине волны, что делает ее предпочтительной и более интересной с точки зрения дальнейшего практического применения серии $\mathrm{Sr}_{2} \mathrm{La}_{8-x} \mathrm{Tm}_{x}\left(\mathrm{GeO}_{4}\right)_{6} \mathrm{O}_{2}$. Доминирующая полоса при $476 \mathrm{~nm}$ отнесена к переходу ${ }^{4} G_{4} \rightarrow{ }^{6} H_{6}$

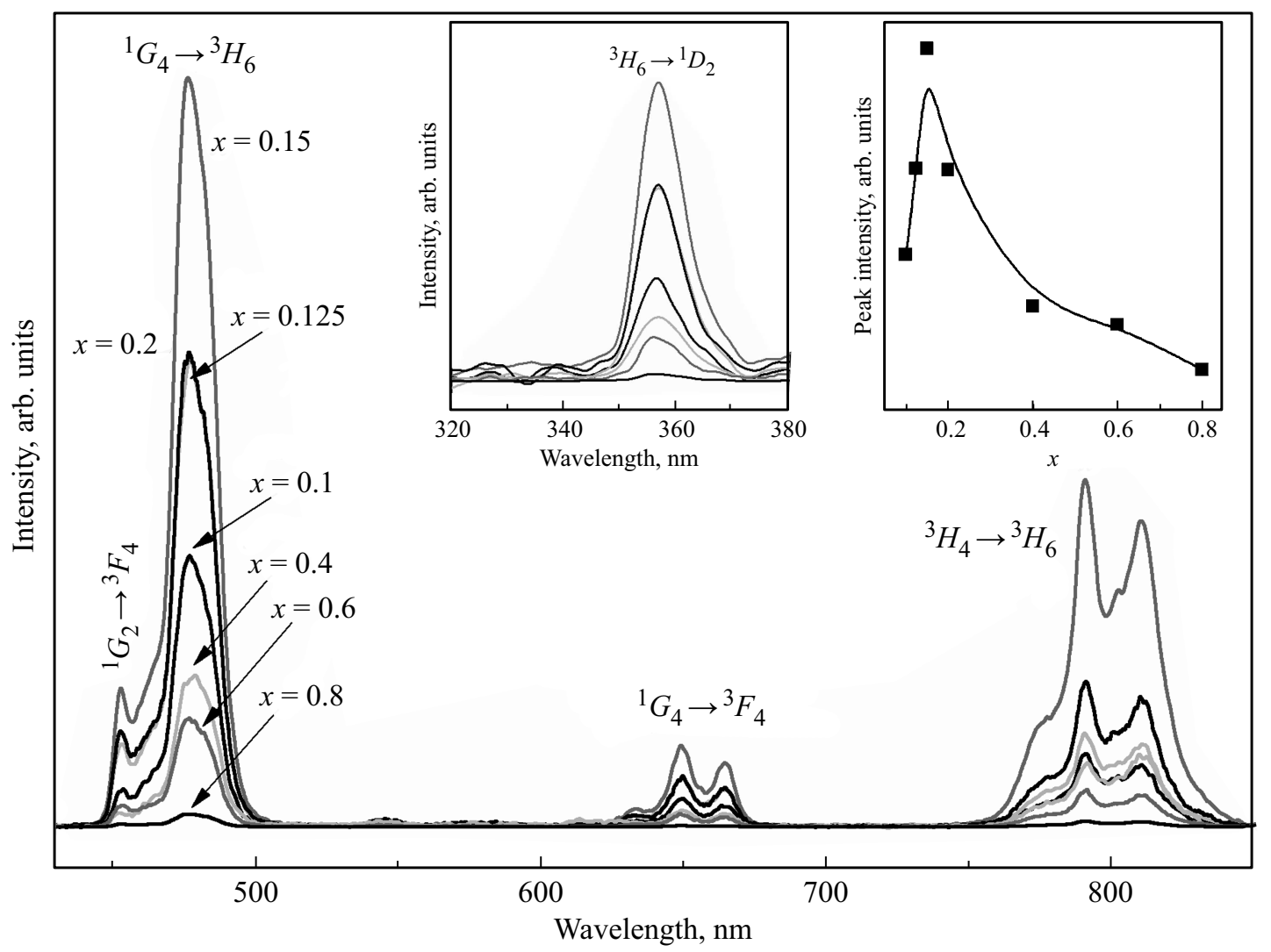

Рис. 1. Спектры люминесценции $\left(\lambda_{\mathrm{ex}}=356 \mathrm{~nm}\right)$ соединений $\mathrm{Sr}_{2} \mathrm{La}_{8-x} \operatorname{Tm}_{x}\left(\mathrm{GeO}_{4}\right)_{6} \mathrm{O}_{2}(x=0.1-1.0)$. Вставки: слева - спектры возбуждения люминесценции $\left(\lambda_{\mathrm{em}}=476 \mathrm{~nm}\right)$ соединений $\mathrm{Sr}_{2} \mathrm{La}_{8-x} \mathrm{Tm}_{x}\left(\mathrm{GeO}_{4}\right)_{6} \mathrm{O}_{2}(x=0.1-1.0)$; справа - концентрационные зависимости интенсивности линии с максимумом при $476 \mathrm{~nm}$ от содержания тулия в $\mathrm{Sr}_{2} \mathrm{La}_{8-x} \mathrm{Tm}_{x}\left(\mathrm{GeO}_{4}\right)_{6} \mathrm{O}_{2}(x=0.1-1.0)$. 


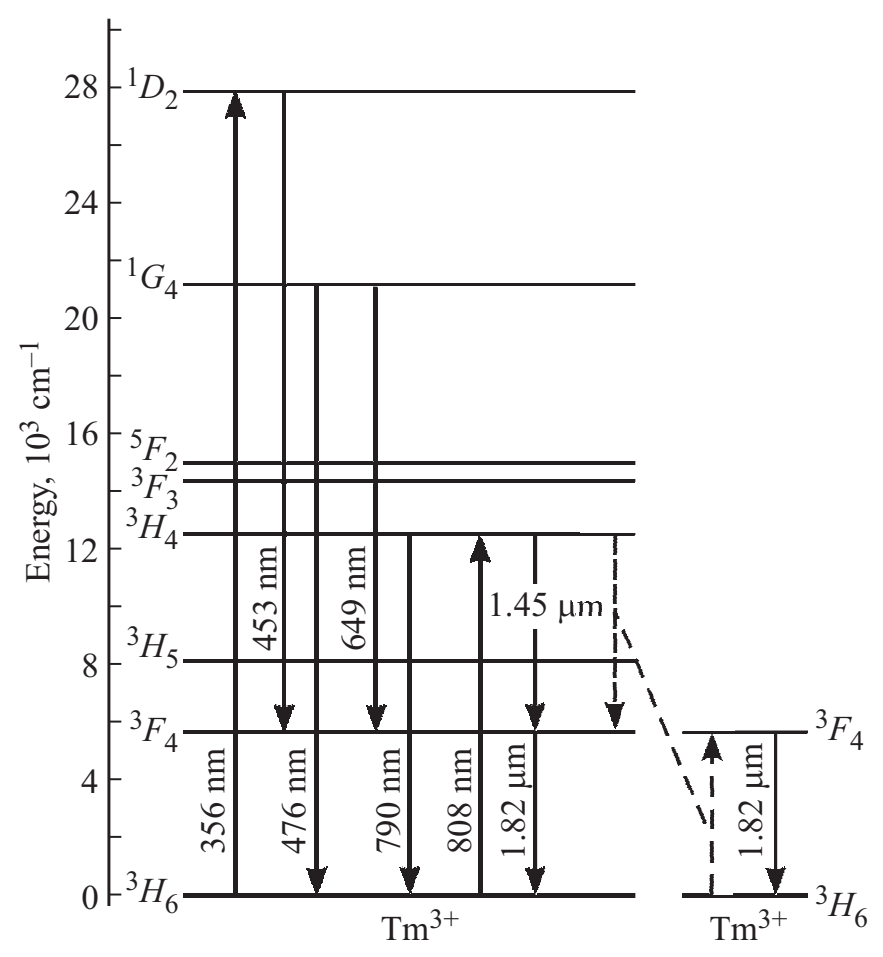

Рис. 2. Схема межуровневых переходов, возбуждения и излучения для ионов $\mathrm{Tm}^{3+}$ в соединениях $\mathrm{Sr}_{2} \mathrm{La}_{8-x} \mathrm{Tm}_{x}\left(\mathrm{GeO}_{4}\right)_{6} \mathrm{O}_{2}$ $(x=0.1-1.0)$. Пунктирными линиями изображены процессы кросс-релаксации; непрерывными линиями - излучательные процессы и процессы поглощения энергии основным состоянием.

в ионах $\mathrm{Tm}^{3+}$. Менее интенсивные линии с максимумом $453 \mathrm{~nm}$ и в диапазонах длин волн $625-675 \mathrm{~nm}$ и 760-830 пm относятся к переходам ${ }^{1} D_{2} \rightarrow{ }^{3} F_{4},{ }^{1} G_{4} \rightarrow{ }^{3} F_{4}$ и ${ }^{3} H_{4} \rightarrow{ }^{3} H_{6}$, соответственно. Максимум фотолюминесценции в серии $\mathrm{Sr}_{2} \mathrm{La}_{8-x} \mathrm{Tm}_{x}\left(\mathrm{GeO}_{4}\right)_{6} \mathrm{O}_{2}$ наблюдается при $x=0.15$. При больших концентрациях тулия интенсивность излучения резко уменьшается вследствие концентрационного тушения (вставка к рис. 1). Схематическое представление процессов возбуждения и релаксации ионов $\mathrm{Tm}^{3+}$, возникающих в апатитах $\mathrm{Sr}_{2} \mathrm{La}_{8-x} \mathrm{Tm}_{x}\left(\mathrm{GeO}_{4}\right)_{6} \mathrm{O}_{2}(x=0.1-1.0)$ при возбуждении длинами волн $356 \mathrm{~nm}$ и $808 \mathrm{~nm}$, показаны на рис. 2.

Координаты цветности, коррелированная цветовая температура и чистота цвета в сравнении с Международным стандартом CIE 1931 года, применяющиеся в качестве характеристик твердотельных осветительных приборов, рассчитаны по спектрам люминесценции соединений $\mathrm{Sr}_{2} \mathrm{La}_{8-x} \mathrm{Tm}_{x}\left(\mathrm{GeO}_{4}\right)_{6} \mathrm{O}_{2}$ при возбуждении длиной волны $356 \mathrm{~nm}$. Координаты цветности $(x, y)$ и чистота цвета для германата $\mathrm{Sr}_{2} \mathrm{La}_{8}\left(\mathrm{GeO}_{4}\right)_{6} \mathrm{O}_{2}: \mathrm{Tm}^{3+}$ с $x$ равным 0.15 составляют $(0.14,0.12)$ и $90 \%$, соответственно, и близки к значениям для коммерчески доступных люминофоров голубого свечения [15]. Коррелированная цветовая температура для германата $\mathrm{Sr}_{2} \mathrm{La}_{7.85} \mathrm{Tm}_{0.15}\left(\mathrm{GeO}_{4}\right)_{6} \mathrm{O}_{2}$, рассчитанная с использова- нием метода, предложенного Мс Саmу [32] составляет $4596 \mathrm{~K}$.

Спектры люминесценции твердых растворов $\mathrm{Sr}_{2} \mathrm{La}_{8-x} \mathrm{Tm}_{x}\left(\mathrm{GeO}_{4}\right)_{6} \mathrm{O}_{2}(x=0.1-1.0)$, измеренные при возбуждении излучением лазерного диода с длиной волны $808 \mathrm{~nm}$, представлены на рис. 3, $a$. Спектры содержат две широкие эмиссионные полосы с максимумами при $1.45 \mu \mathrm{m}$ и $1.82 \mu \mathrm{m}$, соответствующие последовательным переходам ${ }^{3} H_{4} \rightarrow{ }^{3} F_{4}$ и ${ }^{3} F_{4} \rightarrow{ }^{3} H_{6} \quad$ в ионах $\mathrm{Tm}^{3+}$. Максимум интенсивности люминесценции в германатах $\mathrm{Sr}_{2} \mathrm{La}_{8-x} \mathrm{Tm}_{x}\left(\mathrm{GeO}_{4}\right)_{6} \mathrm{O}_{2}(x=0.1-1.0)$ наблюдается для состава с $x=0.4$, т.е. 0.95 at.\%, при более высоких концентрациях $\mathrm{Tm}^{3+}$ интегральная интенсивность всех ИК-полос резко уменьшается (рис. 3,b). Образец с наибольшим содержанием активатора, $x=1.0$ (2.4 at.\%), демонстрирует примерно в 7 раз меньшую эмиссию по сравнению с максимальным по интенсивности составом.

Критическое расстояние $\left(R_{c}\right)$ переноса энергии между соседними ионами лантаноидов можно оценить по уравнению, предложенному Blasse [33]. Учитывая, что объем элементарной ячейки для соединения с критической концентрацией $(x=0.4)$ равен $618.40 \AA^{3}$, критическое расстояние $R_{c}$ составляет $14.3 \AA$. Обменное взаимодействие является преобладающим при малом расстоянии между ионами, не превышающем $5 \AA$ [33-35] и, следовательно, мультипольное взаимодействие является основным механизмом концентрационного тушения в германатах $\mathrm{Sr}_{2} \mathrm{La}_{8-x} \mathrm{Tm}_{x}\left(\mathrm{GeO}_{4}\right)_{6} \mathrm{O}_{2}$.

Тип взаимодействия ионов в процессе концентрационного тушения может быть определен согласно теоретическому описанию взаимосвязи между интенсивностью люминесценции и концентрацией допанта [36]: $I / x=k\left[1+\beta(x)^{\theta / 3}\right]^{-1}$, где $I / x-$ интенсивность эмиссии $(I)$ при концентрации активатора $(x), k$ и $\beta-$ постоянные, $\theta$ - постоянная мультипольного взаимодействия, равная 6,8 или 10 для диполь-дипольного, диполь-квадрупольного и квадруполь-квадрупольного взаимодействия, соответственно. Наклон линейной аппроксимации на кривой зависимости $\lg (I / x)$ от $\lg (x)$ позволяет рассчитать значение $\theta / 3$. Полученное значение $\theta$ составляет 9.42 (рис. 3,c) и близко к 10. Таким образом, доминирующим взаимодействием ионов $\mathrm{Tm}^{3+}$ в германатах $\mathrm{Sr}_{2} \mathrm{La}_{8-x} \mathrm{Tm}_{x}\left(\mathrm{GeO}_{4}\right)_{6} \mathrm{O}_{2}$ в процессе концентрационного тушения является квадрупольквадрупольное взаимодействие.

В зависимости от концентрации тулия интенсивность линии с максимумом при $1.82 \mu \mathrm{m}$ существенно меняется (рис. $3, b$ ), и соотношение эмиссионных линий в спектральных диапазонах $1.35-1.56 \mathrm{~nm}$ и $1.56-2.10 \mu \mathrm{m}$ при максимальной концентрации $(x=1.0)$ превышает в более чем 3 раза значения, полученные для минимальной концентрации тулия $(x=0.1)$. Изменение соотношения между интегральными интенсивностями данных ИК-линий с ростом содержания ионов $\mathrm{Tm}^{3+}$ обычно объясняется увеличением вероятности протекания кроссрелаксационного процесса, способствующего заполнению ${ }^{3} F_{4}$ состояния, который также находит отражение 

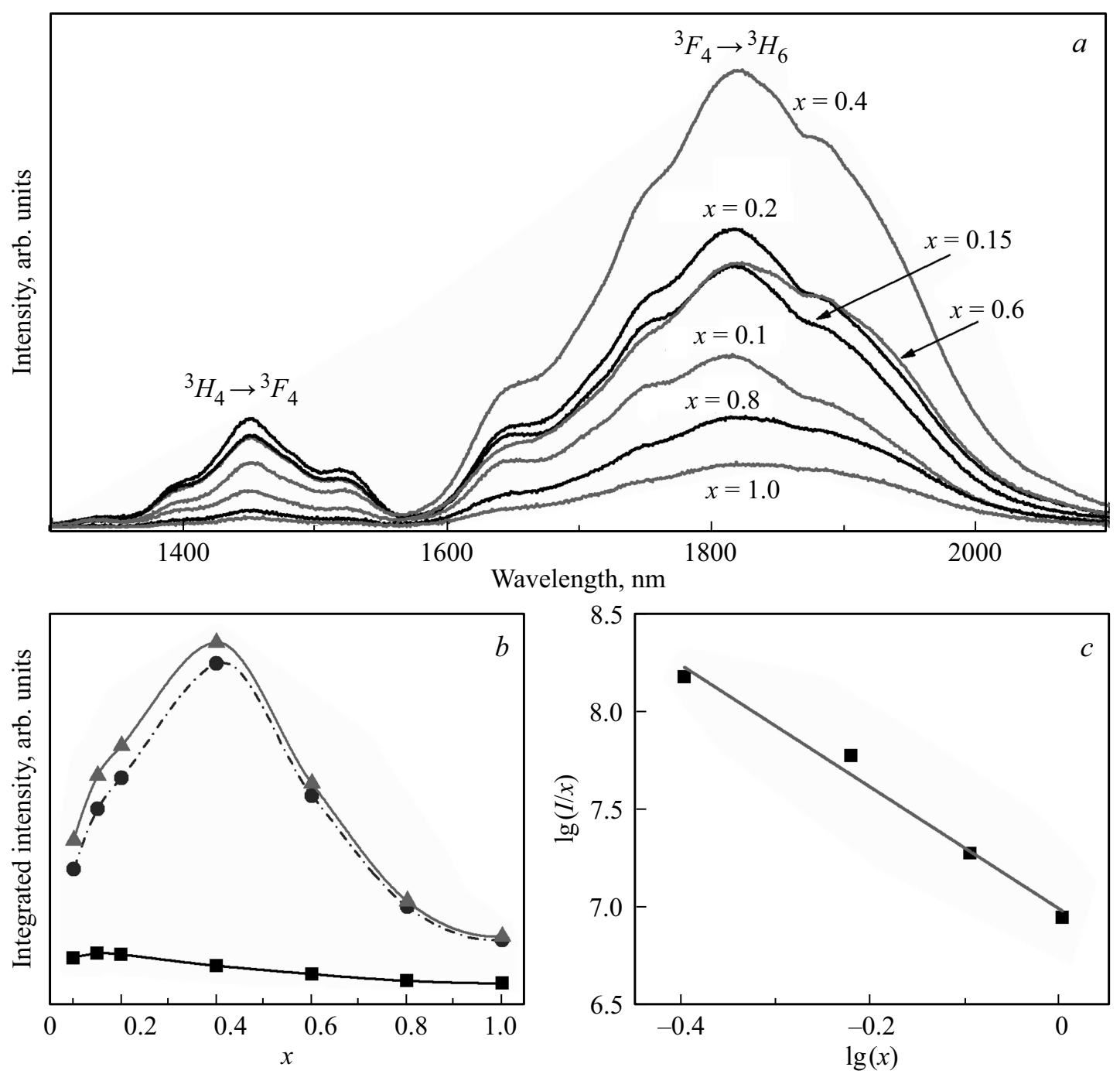

Рис. 3. Спектры люминесценции $\left(\lambda_{\mathrm{ex}}=808 \mathrm{~nm}\right)$ соединений $\mathrm{Sr}_{2} \mathrm{La}_{8-x} \mathrm{Tm}_{x}\left(\mathrm{GeO}_{4}\right)_{6} \mathrm{O}_{2}(x=0.1-1.0)$ (a), концентрационные зависимости интегральной интенсивности (сплошная линия) и интенсивности линии с максимумами при $1.45 \mu \mathrm{m}$ (штриховая линия) и $1.82 \mu \mathrm{m}$ (штрихпунктирная линия) от содержания тулия в $\mathrm{Sr}_{2} \mathrm{La}_{8-x} \mathrm{Tm}_{x}\left(\mathrm{GeO}_{4}\right)_{6} \mathrm{O}_{2}(b)$, зависимость $\lg (I / x)$ от $\lg (x)$ для $\mathrm{Sr}_{2} \mathrm{La}_{8-x} \operatorname{Tm}_{x}\left(\mathrm{GeO}_{4}\right)_{6} \mathrm{O}_{2}(x=0.4-1.0)(c)$.

в изменении величин времени жизни в зависимости от концентрации тулия $[4,8]$. Кроме того, отметим, что и профиль линии с максимумом при $1.82 \mu \mathrm{m}$, отнесенной к сверхчувствительному переходу ${ }^{3} F_{4} \rightarrow{ }_{3} H_{6}$ изменяется при увеличении концентрации $\mathrm{Tm}^{3+}$ вследствие перераспределения компонент на коротковолновом и длинноволновом крыльях полосы (рис. 4). Так, наблюдаемые при малых концентрациях две ярко выраженные компоненты с максимумами при $1.64 \mu \mathrm{m}$ и $1.75 \mu \mathrm{m}$ становятся менее интенсивными при увеличении концентрации тулия, в то время как интенсивность компоненты с максимумом при $1.89 \mu \mathrm{m}$ существенно увеличивается. Рассматриваемые изменения профиля линии люминесценции и соотношения компонент вероятно связаны с перераспределением ионов тулия между $4 f$ и $6 h$ позициями в $\mathrm{Sr}_{2} \mathrm{La}_{8-x} \mathrm{Tm}_{x}\left(\mathrm{GeO}_{4}\right)_{6} \mathrm{O}_{2}$ и формированием двух типов оптических центров. По всей видимости, в край- них составах серии наблюдается преобладание линий от одного из оптических центров тулия, обусловленного его наиболее эффективным возбуждением.

Кинетические кривые затухания люминесценции, измеренные для возбужденных уровней ${ }^{3} H_{4}$ и ${ }^{3} F_{4}$ ионов $\mathrm{Tm}^{3+}$ допированных $\mathrm{Sr}_{2} \mathrm{La}_{8-x} \mathrm{Tm}_{x}\left(\mathrm{GeO}_{4}\right)_{6} \mathrm{O}_{2}$ $(x=0.1-1.0)$ имеют двухэкспоненциальный вид распада (рис. 5) и были аппроксимированы суммой двух экспоненциальных функций. Двухэкспоненциальный профиль кривых затухания люминесценции может быть связан с существованием в кристаллической решетке $\mathrm{Sr}_{2} \mathrm{La}_{8-x} \mathrm{Tm}_{x}\left(\mathrm{GeO}_{4}\right)_{6} \mathrm{O}_{2}$ двух позиций с различным локальным окружением, занятых ионами-активаторами, а также с возникновением кросс-релаксационного процесса. Полученные значения величин времени жизни ${ }^{3} \mathrm{H}_{4}$ и ${ }^{3} F_{4}$ состояний иона $\mathrm{Tm}^{3+}$ изменяются в диапазонах $14-188 \mu \mathrm{s}$ и $89-575 \mu \mathrm{s}$, соответственно, и являются 


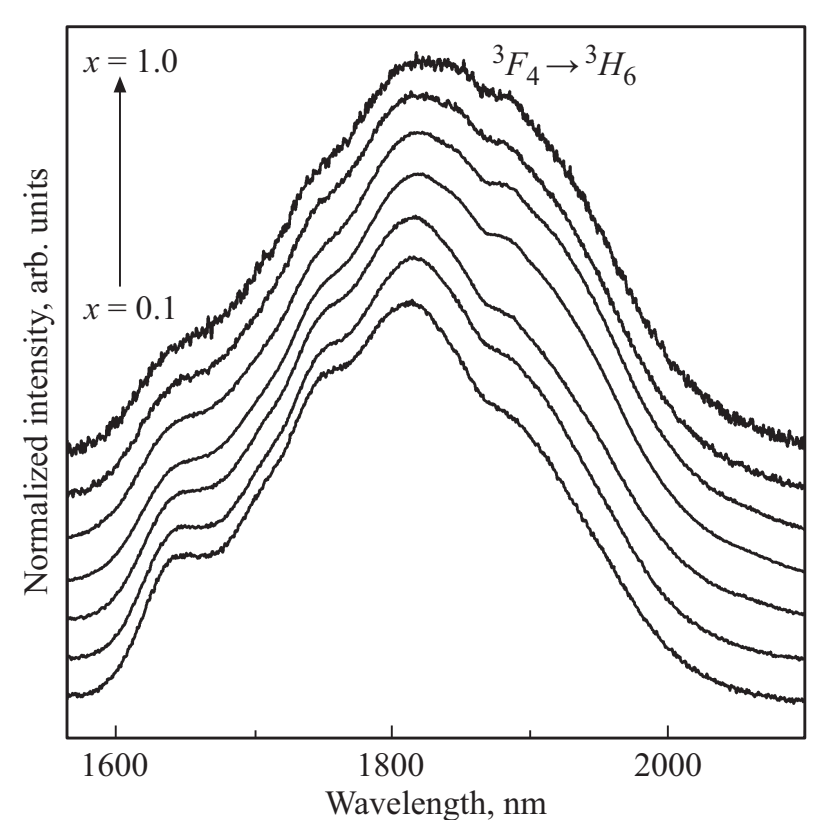

Рис. 4. Нормированные по амплитуде спектры люминесценции $\left(\lambda_{\mathrm{ex}}=808 \mathrm{~nm}\right)$ соединений $\mathrm{Sr}_{2} \mathrm{La}_{8-x} \mathrm{Tm}_{x}\left(\mathrm{GeO}_{4}\right)_{6} \mathrm{O}_{2}$ $(x=0.1-1.0)$ в диапазоне $1570-2100 \mathrm{~nm}$.

типичными для соединений, допированных $\mathrm{Tm}^{3+}[4,6,8]$. С увеличением содержания допанта наблюдается закономерная тенденция к уменьшению времени жизни, вы- званная процессом концентрационного тушения. Более того эффективное протекания безызлучательного кроссрелаксационного процесса ${ }^{3} H_{4}+{ }^{3} H_{6} \rightarrow{ }^{3} F_{4}+{ }^{3} F_{4}$ между ионами $\mathrm{Tm}^{3+}$ подтверждается неравномерным уменьшением значений времени жизни для возбужденных состояний ${ }^{3} \mathrm{H}_{4}$ и ${ }^{3} \mathrm{~F}_{4}$.

Температура тушения люминесценции является одним из важнейших технологических параметров для люминофоров, которая определяет возможности применения материалов в большинстве оптических устройств, в частности, содержащих мощные источники возбуждения. Спектры люминесценции германата $\mathrm{Sr}_{2} \mathrm{La}_{7.6} \mathrm{Tm}_{0.4}\left(\mathrm{GeO}_{4}\right)_{6} \mathrm{O}_{2}$ с максимальной интенсивностью эмиссии в коротковолновой ИК-области, измеренные в широком температурном интервале приведены на рис. 6,a. Постепенный нагрев образца до $220^{\circ} \mathrm{C}$ сопровождается равномерным увеличением интенсивности всех эмиссионных полос, и температурное тушение люминесценции, традиционно приписываемое увеличению скорости безызлучательной релаксации, не наблюдается (рис. $6, b$ ). Обычно рост интенсивности при повышенных температурах связывают с процессами переноса энергии между ионами, который наблюдается, например, в апатите $\mathrm{NaLa}_{9}\left(\mathrm{GeO}_{4}\right)_{6} \mathrm{O}_{2}$, содопированном $\mathrm{Tm}^{3+}$ и $\mathrm{Dy}^{3+}[19]$, или с термически активированной безызлучательной релаксацией более высоких уровней энергии, как в случае легированных $\mathrm{Tm}^{3+}$ теллуритных стекол [4]. Однако оба этих процесса приводят к неравномерному усилению одной линии
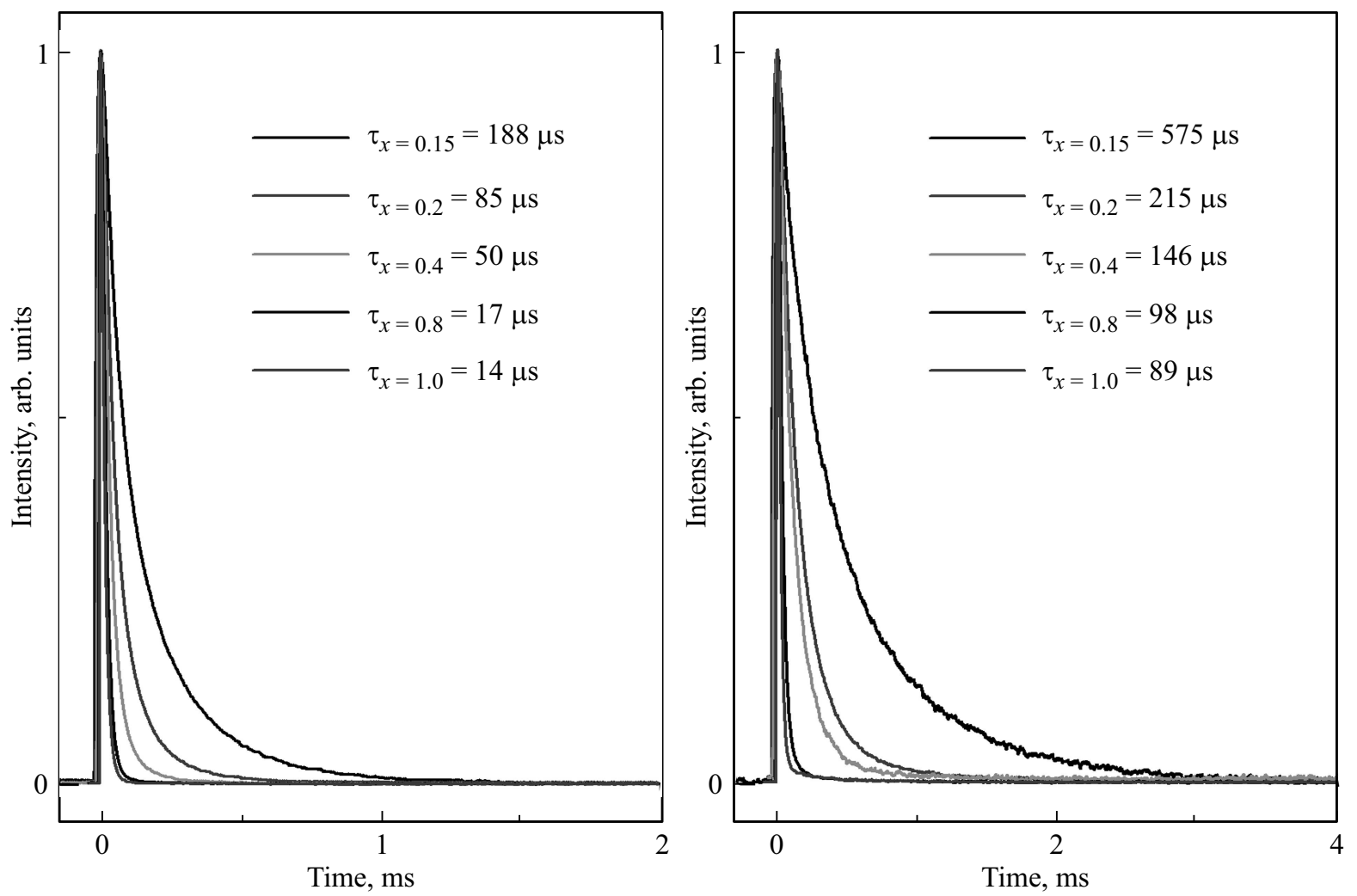

Рис. 5. Кривые затухания люминесценции германатов $\mathrm{Sr}_{2} \mathrm{La}_{8-x} \mathrm{Tm}_{x}\left(\mathrm{GeO}_{4}\right)_{6} \mathrm{O}_{2}(x=0.15-1.0)$, измеренные для возбужденных уровней ${ }^{3} H_{4}(a)$ и ${ }^{3} F_{4}(b)$ иона $\mathrm{Tm}^{3+}$. 

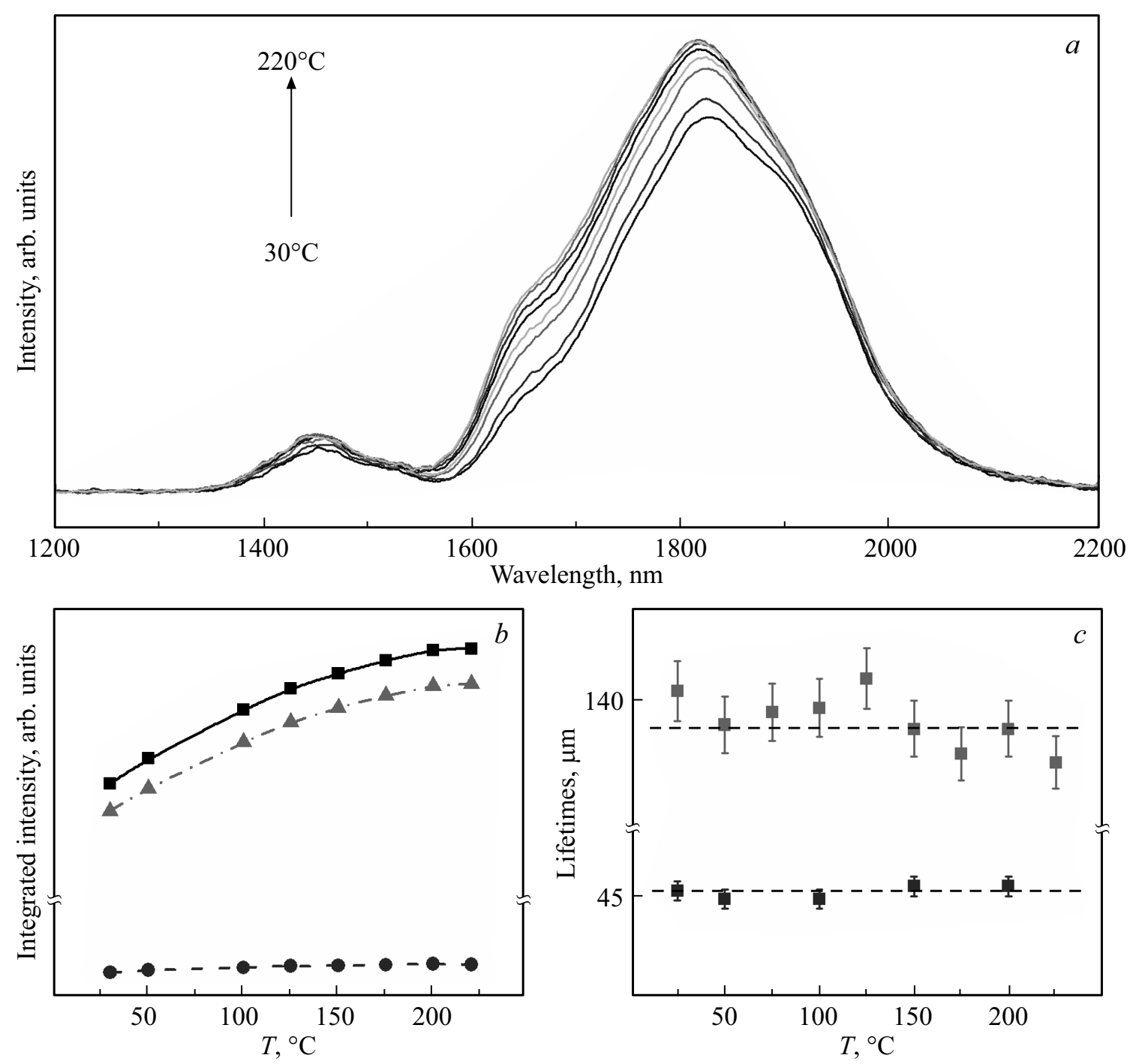

Рис. 6. Спектры люминесценции $\left(\lambda_{\mathrm{ex}}=808 \mathrm{~nm}\right)$ соединения $\mathrm{Sr}_{2} \mathrm{La}_{7.6} \mathrm{Tm}_{0.4}\left(\mathrm{GeO}_{4}\right)_{6} \mathrm{O}_{2}$, зарегистрированные в интервале температур от $30^{\circ} \mathrm{C}$ до $220^{\circ} \mathrm{C}(a)$, температурные зависимости интегральной интенсивности (сплошная линия) и интенсивностей линий с максимумами при $1.45 \mu \mathrm{m}$ (штриховая линия) и $1.82 \mu \mathrm{m}$ (штрихпунктирная линия) (a) и температурные зависимости величин времени жизни уровней ${ }^{3} H_{4}$ (штриховая линия) и ${ }^{3} F_{4}$ (штрихпунктирная линия) иона $\mathrm{Tm}^{3+}(c)$.

при сохранении или уменьшении интенсивности другой линии с увеличением температуры. Равномерный рост интегральных интенсивностей всех линий может быть объяснен изменением поглощения возбуждающего излучения в зависимости от температуры вследствие перераспределения по различным компонентам кристаллического поля основного состояния [37]. В случае германатов $\mathrm{Sr}_{2} \mathrm{La}_{8}\left(\mathrm{GeO}_{4}\right)_{6} \mathrm{O}_{2}: \mathrm{Tm}^{3+}$ можно ожидать расщепление состояния ${ }^{3} \mathrm{H}_{6}$ на штарковские уровни, предполагаемые для $C_{3}$ и $C_{s}$ симметрий, соответствующие двум кристаллическим центрам $4 f$ и $6 h$, занятым ионами $\mathrm{Tm}^{3+}$. С повышением температуры происходит заселение более высоких штарковских уровней состояния ${ }^{3} H_{6}$ и смещение максимума поглощения в сторону более низких энергий. Наиболее точное определение температуры тушения люминесценции соединений воз- можно только при учете температурной зависимости времени затухания люминесценции, представленной на рис. 6,c. Для оптимального по концентрации состава $\mathrm{Sr}_{2} \mathrm{La}_{7.6} \mathrm{Tm}_{0.4}\left(\mathrm{GeO}_{4}\right)_{6} \mathrm{O}_{2}$ значения времени жизни возбужденных уровней ${ }^{3} H_{4}$ и ${ }^{3} F_{4}$ иона $\mathrm{Tm}^{3+}$ не изменяется во всем зарегистрированном температурном интервале, и подтверждает отсутствие температурного тушения люминесценции до $220^{\circ} \mathrm{C}$. Следовательно, тушение люминесценции для германатов допированных $\mathrm{Tm}^{3+}$ наблюдается при температуре, превышающей требуемые для современных люминофоров рабочие значения. Таким образом, превосходная термическая стабильность и интенсивность эмиссии позволяет рассматривать апатит $\mathrm{Sr}_{2} \mathrm{La}_{8}\left(\mathrm{GeO}_{4}\right)_{6} \mathrm{O}_{2}: \mathrm{Tm}^{3+}$ в качестве перспективного люминофора коротковолнового ИК-диапазона. 


\section{4. Заключение}

Германаты $\mathrm{Sr}_{2} \mathrm{La}_{8-x} \operatorname{Tm}_{x}\left(\mathrm{GeO}_{4}\right)_{6} \mathrm{O}_{2}(x=0.1-1.0)$ со структурой апатита получены с использованием твердофазного метода. Согласно рентгеноструктурному анализу, соединения кристаллизуются в гексагональной системе (пространственная группа $P 6_{3} / m, Z=1$ ). Установлены основные закономерности изменения люминесцентных свойств твердых растворов $\mathrm{Sr}_{2} \mathrm{La}_{8}\left(\mathrm{GeO}_{4}\right)_{6} \mathrm{O}_{2}: \mathrm{Tm}^{3+}$ в видимом и коротковолновом инфракрасном диапазоне при возбуждении длинами волн $356 \mathrm{~nm}$ и $808 \mathrm{~nm}$. На спектрах фотолюминесценции германатов в диапазоне длин волн $430-850 \mathrm{~nm}$ доминирующая линия с максимумом при $476 \mathrm{~nm}$ обусловлена переходом ${ }^{1} G_{4} \rightarrow{ }^{3} H_{6}$ в ионах $\mathrm{Tm}^{3+}$. Координаты цветности и коррелированная цветовая температура германата $\mathrm{Sr}_{2} \mathrm{La}_{7.85} \mathrm{Tm}_{0.15}\left(\mathrm{GeO}_{4}\right)_{6} \mathrm{O}_{2}$, проявляющего наиболее интенсивное излучение в видимом диапазоне, близки к значениям для коммерчески доступных люминофоров голубого свечения. Спектры люминесценции германатов в ИК-спектральном диапазоне состоят из двух широких полос с максимумами при $1.45 \mu \mathrm{m}$ и $1.82 \mu \mathrm{m}$, которые соответствуют каскадным переходам ${ }^{3} H_{4} \rightarrow{ }^{3} F_{4}$ и ${ }^{3} F_{4} \rightarrow{ }^{3} H_{6}$ в ионах $\mathrm{Tm}^{3+}$. С увеличением концентрациях тулия в германатах наблюдается усиление $1.82 \mu \mathrm{m}$ полосы вследствие эффективного протекания безызлучательного кросс-релаксационного процесса. Для германата $\mathrm{Sr}_{2} \mathrm{La}_{7.6} \mathrm{Tm}_{0.4}\left(\mathrm{GeO}_{4}\right)_{6} \mathrm{O}_{2}$ с максимальной интенсивностью эмиссии в коротковолновой ИК-области температурное тушение люминесценции в интервале $30-220^{\circ} \mathrm{C}$ не наблюдается. Полученные результаты указывают на широкие возможности использования германиевых соединений со структурой апатита в качестве потенциальных люминесцентных материалов видимого и коротковолнового ИК-спектральных диапазонов.

\section{Финансирование работы}

Работа выполнена в рамках проекта Российского научного фонда № 16-13-10111.

\section{Конфликт интересов}

Авторы заявляют, что у них нет конфликта интересов.

\section{Список литературы}

[1] F.K. Tittel, D. Richter, A. Fried. In: Solid-State MidInfrared Laser Sources / Ed. I.T. Sorokina, K.L. Vodopyanov. SpringerVerlag Berlin Heidelberg Ltd, Berlin (2003). P. 458.

[2] B. Jean, T. Bende, A. Fried. In: Solid-State MidInfrared Laser Sources / Ed. I.T. Sorokina, K.L. Vodopyanov. SpringerVerlag Berlin Heidelberg Ltd, Berlin. (2003). P. 530.

[3] S.D. Jackson. Nature Photonics 6, 423 (2012).

[4] S. Tanabe. Proc. SPIE 85, 4282 (2001).

[5] J.Q. Hong, L.H. Zhang, M. Xu, Y. Hang. Opt. Mater. Express 6, 1444 (2016)
[6] R. Chen, Y. Tian, B. Li, X. Jing, J. Zhang, S. Xu, H. Eckert, X. Zhang. Photon. Res. 4, 214 (2016).

[7] M.C. Falconi, D. Laneve, F. Prudenzano. Fibers. 5, 23 (2017).

[8] H. Cankaya, A. Tolga Gorgulu, A. Kurt, A. Speghini, M. Bettinelli, A. Sennaroglu. Appl. Sci. 8, 333 (2018).

[9] T.J. White, D. ZhiLi. Acta Cryst. B 59, 1 (2003).

[10] M. Wierzbicka-Wieczorek, M. Göckeritz, U. Kolitsch, C. Lenz, G. Giester. Eur. J. Inorg. Chem. 6, 948 (2015).

[11] P.R. Slater, J.E.H. Sansom, J.R. Tolchard. Chem. Rec. 4, 373 (2004).

[12] Y. Higuchi, M. Sugawara, K. Onishi, M. Sakamoto, S. Nakayama. Ceram. Int. 36, 955 (2010).

[13] C. Piccirillo, P.M.L. Castro, J. Environ. Manage 193, 79 (2017).

[14] I.A. Neacsu, A.E. Stoica, B.S. Vasile, E. Andronescu. Nanomater. 9, 239 (2019).

[15] G.S.R. Raju, J.Y. Park, H.C. Jung, E. Pavitra, B.K. Moon, J.H. Jeong, J.S. Yu, J.H. Kim, H. Choi. J. Alloys Compd. 509, 7537 (2011).

[16] M. Que, Z. Ci, Y. Wang, G. Zhu, Y. Shi, S. Xin, J. Lumin. 144, 64 (2013).

[17] D. Kim, D. Park, N. Oh, J. Kim, E.D. Jeong, S.J. Kim, S. Kim, J.-C. Park. Inorg. Chem. 54, 1325 (2014).

[18] Y.I. Jeon, L.K. Bharat, J.S. Yu. J. Lumin. 166, 93, (2015).

[19] Y. Cao, X. Ding, Y. Wang. J. Am. Ceram. Soc. 99, 3696 (2016).

[20] N. Liu, L. Mei, L. Liao, J. Fu, D. Yang. Sci. Rep. 9, 15509 (2019).

[21] K.B. Steinbruegge, T. Henningsen, R.H. Hopkins, R. Mazelsky, N.T. Melamed, E.P. Riedel, G.W.R. May. Appl. Opt. 11, 999 (1972).

[22] P. Raybaut, F. Druon, F. Balembois, P.G.R. Gaume, B. Viana, D. Vivien. Opt. Lett. 28, 2195 (2003).

[23] H.K. Juwhari, M.H. Kailani, B.I. Lahlouh, S.A. Abedrabbo, K.A. Saleh, W.B. White. Mater. Lett. 87, 80 (2012).

[24] Y.V. Baklanova, O.A. Lipina, A.N. Enyashin, L.L. Surat, A.P. Tyutyunnik, N.V. Tarakina, A. Dominic Fortes, A.Yu. Chufarov, E.V. Gorbatova, V.G. Zubkov. Dalton Trans. 47, 14041 (2018).

[25] L. Wu, Y. Zhang, M. Gui, P. Lu, L. Zhao, S. Tian, Y. Kong, J. Xu. Mater. Chem. 22, 6463 (2012).

[26] C. Wang, J. Jiang, S. Xin, Y. Shi, G. Zhu. J. Lumin. 214, 116521 (2019).

[27] B.H. Toby. J. Appl. Crystallogr. 34, 210 (2001).

[28] A.C. Larson, R.B. Von Dreele. General Structure Analysis System (GSAS). Los Alamos, NM (2004). Los Alamos National Laboratory Report LAUR 86-748.

[29] R.D. Shannon, C.T. Prewitt. Acta Crystallogr. B 25, 925 (1969).

[30] Y.Q. Jia. J. Solid State Chem. 95, 184 (1991).

[31] M. Stefanski, K. Grzeszkiewicz, M. Ptak, D. Hreniak, W. Strek. J. Chem. Phys. 150, 094706 (2019).

[32] S. Mc Camy. Color Res. Appl. 17, 142 (1992).

[33] G. Blasse. Phys. Lett. A 28, 444 (1968).

[34] D.L. Dexter. J. Chem. Phys. 21, 836 (1953).

[35] D.L. Dexter, L. Shulman. J. Chem. Phys. 22, 1063 (1954).

[36] L.G. Van Uitert. J. Electrochem. Soc. 114, 1048 (1967).

[37] V. Bachmann, C. Ronda, A. Meijerink. Chem. Mater. 21, 2077 (2009).

Редактор К.В. Емщев 